\title{
Fast non-iterative methods for defect identification
}

\author{
Marc Bonnet* — Bojan B. Guzina*** - Nicolas Nemitz*,** \\ * Laboratoire de Mécanique des Solides (CNRS UMR 7649) \\ Ecole Polytechnique, 91128 Palaiseau cedex, France \\ bonnet@lms.polytechnique.fr \\ ** Laboratoire Jacques-Louis Lions \\ Université Pierre et Marie Curie \\ 75252 Paris cedex 05, France \\ nemitz@ann.jussieu.fr \\ *** Department of Civil Engineering, University of Minnesota \\ 500 Pillsbury Drive S.E., Minneapolis, MN 55455, USA \\ guzina@wave.ce.umn.edu
}

ABSTRACT. This communication summarizes recent investigations on the identification of defects (cavities, inclusions) of unknown geometry and topology by means of the concept of topological sensitivity. This approach leads to the fast computation (equivalent to performing a few direct solutions), by means of ordinary numerical solution methods such as the BEM (used here), the FEM or the FDM, of defect indicator functions. Substantial further acceleration is obtained by using fast multipole accelerated BEMs. Possibilities afforded by this approach are demonstrated on numerical examples. The paper concludes with a discussion of further research on theoretical and numerical issues.

RÉSUMÉ. Cette communication présente une synthèse de travaux consacrés à l'identification de défauts (cavités, inclusions) de géométrie et topologie a priori inconnus par des approches non itératives reposant sur la notion de sensibilité topologique. Ces méthodes permettent des calculs rapides (coût de l'ordre de quelques calculs directs), par des méthodes numériques ordinaires, de champs indicateurs de défauts, et peuvent être accélérés par la méthode multipôle rapide. Quelques possibilités de ces approches sont illustrées sur des exemples numériques. Des perspectives touchant la théorie et la mise en cuvre numérique sont présentées.

KEYWORDS: inverse problems, topological sensitivity, fast multipole method.

MOTS-CLÉS : problèmes inverses, sensibilité topologique, méthode multipôle rapide.

DOI:10.3166/REMN.17.571-582 @ 2008 Lavoisier, Paris

REMN - 17/2008. Giens 2007, pages 571 to 582 


\section{Introduction}

The reconstruction of obstacles embedded in an acoustic medium is a challenging subject with application to e.g. sonar detection and medical imaging (Lambert et al., 2006; Colton et al., 2000). It is often formulated in terms of the minimization of a cost function featuring the experimental data and (possibly) prior information. Such cost functions are non-convex and exhibit local minima. Global search techniques, e.g. evolutionary algorithms (Michalewicz and Fogel, 2004) or parameter space sampling methods (Tarantola, 2005) require very large numbers of cost function evaluations, and hence are usually prohibitively expensive in the context of wave-based approaches. Hence, traditional minimization methods, or Newton-type algorithms for solving the observation equations, are often preferred, as they may converge within moderate iteration counts for obstacles described in terms of a small number of geometrical parameters. To optimize computational efficiency, such solution techniques are often used in conjunction with shape sensitivity techniques (Bonnet, 1995; Guzina et al., 2003; Farhat et al., 2002) or level-set methods (Litman et al., 1998).

Still, the stand-alone use of gradient-based algorithms for such purposes is not always satisfactory due to their strong dependence on a reliable prior information about the geometry of the hidden object. This has prompted, over the last decade the development of alternative, non-iterative, approaches, which may be used either in isolation or as a preliminary step providing adequate initial guesses to subsequent iterative schemes. Now referred to as "sampling" or "probe" methods (Potthast, 2006), they aim at replacing the usual optimization approach with the definition of a defect indicator function. They include approaches based on linear sampling (Colton and Kirsch, 1996), not pursued here, or on the concept of topological sensitivity (Bonnet and Guzina, 2004; Guzina and Bonnet, 2004), or more generally on small-defect asymptotics (Ammari and Kang, 2004). This communication aims at summarizing contributions we made in this area over the last few years.

\section{Topological sensitivity and inversion}

The concept of topological derivative first appeared in (Eschenauer et al., 1994; Schumacher, 1995) in the context of topological optimization of mechanical structures, allowing to define algorithms where "excess" material is iteratively removed until a satisfactory shape and topology is reached. Following investigations by e.g. Sokolowski and Zochowski (1999) and Garreau et al. (2001) for the Laplace equation or Samet et al. (2004) and Pommier and Samet (2005) for the Helmholtz and Maxwell equations, we study here the usefulness of the topological sensitivity field as a defect indicator function. Our work addresses only 3-D configurations, but 2-D cases are considered by e.g. Feijóo (2004) or Gallego and Rus (2004).

This approach rests upon quantifying the sensitivity of the cost function to the nucleation of an object, of specified physical properties and small characteristic size $\varepsilon$, at a specified location $\boldsymbol{x}_{\mathrm{s}}$ in the reference medium. Letting $J\left(\varepsilon, \boldsymbol{x}_{\mathrm{s}}\right)$ denote the 
value achieved by the featured cost function of the inverse problem when only the infinitesimal obstacle located at $\boldsymbol{x}_{\mathrm{s}}$ is present, the topological sensitivity $\mathcal{T}_{3}\left(\boldsymbol{x}_{\mathrm{s}}\right)$ of $J$ is (for 3D configurations involving either sound-hard or penetrable obstacles, i.e. such that either Neumann or transmission conditions are assumed on the obstacle surface) defined through the expansion

$$
J\left(\varepsilon, \boldsymbol{x}_{\mathrm{s}}\right)-J\left(0, \boldsymbol{x}_{\mathrm{s}}\right)=\varepsilon^{3} \mathcal{T}_{3}\left(\boldsymbol{x}_{\mathrm{s}}\right)+o\left(\varepsilon^{3}\right)
$$

Note that the value $J\left(0, \boldsymbol{x}_{\mathrm{s}}\right)$ of $J$ for the obstacle-free reference medium does not actually depend on $\boldsymbol{x}_{\mathrm{s}}$. The topological sensitivity field $\mathcal{T}_{3}\left(\boldsymbol{x}_{\mathrm{s}}\right)$ is then used as a defect indicator function. Heuristically, regions in the probed medium in which the nucleation of a small defect induces the strongest decrease of $J$ are expected to be those where the field $\mathcal{T}_{3}\left(\boldsymbol{x}_{\mathrm{s}}\right)$ attains its most pronounced negative values. A simple approach then consists in computing the field $\mathcal{T}_{3}\left(\boldsymbol{x}_{\mathrm{s}}\right)$ over a region of space susceptible of housing defects and look for locations where $\mathcal{T}_{3}$ attains its lowest negative values.

The topological sensitivity field $\mathcal{T}_{3}\left(\boldsymbol{x}_{\mathrm{s}}\right)$ may be given an explicit expression in terms of the Green's function associated with the reference domain, its physical properties, and the type of boundary conditions assumed for the external boundary. In practice, the relevant Green's function is not known in explicit form. Another approach, better suited to numerical computations, consists in expressing $\mathcal{T}_{3}\left(\boldsymbol{x}_{\mathrm{s}}\right)$ in terms of the free field $u$ (response of the reference, i.e. defect-free, medium to the probing excitation) and the adjoint solution $\hat{u}$ (induced by an adjoint load defined in terms of the cost function). For example, the topological sensitivity field $\mathcal{T}_{3}\left(\boldsymbol{x}_{\mathrm{s}}\right)$ corresponding to the nucleation of a small sound-hard spherical scatterer, under time-harmonic conditions, in a linearly acoustic reference medium characterized by wave number $k$ is found (e.g. Nemitz and Bonnet (2007)) to be given by

$$
\mathcal{T}_{3}\left(\boldsymbol{x}_{\mathrm{s}}\right)=\frac{4 \pi}{3}\left[\frac{3}{2} \nabla u . \nabla \hat{u}-k^{2} u \hat{u}\right]\left(\boldsymbol{x}_{\mathrm{s}}\right)
$$

Expressions of $\mathcal{T}_{3}\left(\boldsymbol{x}_{\mathrm{s}}\right)$ have also been established for other 3-D configurations. Such results include, for the time-harmonic case, acoustic media containing penetrable obstacles (Guzina and Bonnet, 2006), elastic media with cavities (Guzina and Bonnet, 2004; Bonnet and Guzina, 2004) or elastic inclusions (Guzina and Chikichev, 2007), and electromagnetic media containing penetrable inclusions (Ammari and Kang, 2004; Masmoudi et al., 2005). For time domain analyses, similar formulae have been established for $\mathcal{T}_{3}\left(\boldsymbol{x}_{\mathrm{s}}\right)$, in which bilinear expressions in $(u, \hat{u})$ such as [2] become time convolution products between free and adjoint fields (Bonnet, 2006). It is useful to note that expressions such as [2] are formally similar to sums of a strain energy and a kinetic energy, and thus are well suited to any usual computational approach, such as the finite element method (FEM) or the boundary element method (BEM) used in our numerical experiments to date.

\section{Topological sensitivity and fast multipole method}

When the reference medium is endowed with simple (linear, piecewisehomogeneous) constitutive properties, the topological sensitivity field $\mathcal{T}_{3}\left(\boldsymbol{x}_{\mathrm{s}}\right)$ may be 
formulated using integral representation formulae (Bonnet, 1999a), the free and adjoint fields then being first computed over the external boundary by means of a BEM. This approach is in particular well suited to dealing with unbounded reference media. As probing a 3-D region of space may necessitate large numbers of sampling points $\boldsymbol{x}_{\mathrm{s}}$, such computation may nevertheless entail high CPU and memory requirement, because of the fully-populated nature of the BEM influence matrix. The Fast Multipole Method (FMM) (Greengard and Rokhlin, 1997; Nishimura, 2002; Margonari and Bonnet, 2005; Nemitz, 2006; Chaillat et al., 2007) addresses these concerns, as it provides considerable speedup and memory savings. For a frequency-domain analysis involving $N$ BEM DOFs and $M$ sampling points, computing the field $\mathcal{T}_{3}\left(\boldsymbol{x}_{\mathrm{s}}\right)$ requires $O(N \log N)$ operations per GMRES iteration (for the BEM solution phase) and $O(M \log M)$ operations (subsequent evaluation of $\mathcal{T}_{3}\left(\boldsymbol{x}_{\mathrm{s}}\right)$ at sampling points), whereas the corresponding complexities for a traditional BEM are $O\left(N^{2}\right)$ per solver iteration (or $O\left(N^{3}\right)$ for direct solvers) and $O(M N)$. The FMM allows to handle computational models with $N, M=O\left(10^{6}\right)$ on an ordinary PC.

Numerical example. The above-summarized approach combining the topological sensitivity and the FMM (Nemitz and Bonnet, 2007) is now demonstrated on the following configuration: the bounded acoustic domain is the cube defined by $\Omega=\left\{\left|\xi_{i}\right| \leq 8 a(i=1,2,3)\right\}$, where $a$ is a reference length. A simulated testing configuration is based on 30 experiments, each of which consists in applying a uniform excitation over a small region $S_{q}(1 \leq q \leq 30)$ of the external boundary $S$ and recording the acoustic pressure over all of $S$ (i.e. at all BE mesh nodes). The acoustic excitation is such that the wavelength is $\lambda=3 a$. Each of the six faces of the cubical domain $\Omega$ supports five excitation surfaces $S_{q}$, each a disk of radius $a$. The cost function for the inverse problem is defined by

$$
\mathcal{J}\left(\Omega^{\star}\right)=\frac{1}{2} \sum_{q=1}^{30} \int_{S}\left|u^{\star}-u_{q}^{\mathrm{obs}}\right|^{2} \mathrm{~d} \Gamma_{\xi}
$$

where $u_{q}^{\text {obs }}$ is the data obtained for the $q$-th applied excitation, with $u_{q}^{\text {obs }}=u^{\text {true }}$ in the absence of data noise. The centroid $\boldsymbol{x}^{\text {true }}$ of true scatterer $B^{\text {true }}$ to be identified is located at $\boldsymbol{x}^{\text {true }}=(2 a, 3 a, 2 a)$. To facilitate the graphical interpretation, a thresholded variant $\hat{\mathcal{T}}\left(\boldsymbol{x}_{\mathrm{s}}\right)$ of $\mathcal{T}\left(\boldsymbol{x}_{\mathrm{s}}\right)$ is introduced according to

$$
\hat{\mathcal{T}}_{3}\left(\boldsymbol{x}_{\mathrm{s}}\right)=\mathcal{T}_{3}\left(\boldsymbol{x}_{\mathrm{s}}\right) \quad\left(\mathcal{T}_{3} \leq C \mathcal{T}_{\text {min }}\right), \quad \hat{\mathcal{T}}_{3}\left(\boldsymbol{x}_{\mathrm{s}}\right)=0\left(\mathcal{T}_{3}>C \mathcal{T}_{\text {min }}\right),
$$

with $C=0.25$ used here. The BE meshes used for computing the free field $u$, the adjoint field $\hat{u}$ and the simulated data $u_{q}^{\text {true }}$ are made of three-noded flat triangular elements, arranged in a regular mesh with approximately 15 nodes per wavelength. For the purposes of computing the simulated error-free data $u^{\text {true }}$ for each synthetic experiment, BE meshes of the true scatterer $\Gamma^{\text {true }}$ have been set up as well. Table 1 indicates the numbers of nodes and elements supported by the BE meshes.

The identification of one spherical scatterer of radius $0.4 a$ is considered. The field $\mathcal{T}$ has been computed on the basis of error-free synthetic data, over a sampling 
Table 1. Number of element and DOFs supported by the BE meshes

\begin{tabular}{|l|rr|rr|rr|}
\hline Cube size & \multicolumn{2}{|c|}{ Cube } & \multicolumn{2}{c|}{ Obstacle } & \multicolumn{2}{c|}{ Total } \\
& Elements & nodes & Elements & nodes & Elements & nodes \\
\hline $2 L=16 a$ & 76800 & 38402 & 336 & 170 & 77136 & 38572 \\
$2 L=32 a$ & 307200 & 153602 & 336 & 170 & 307536 & 153772 \\
\hline
\end{tabular}

grid made of $100 \times 100 \times 100$ sampling points located on the vertices of a regular cubic grid, centered at the origin and with grid spacing $\Delta x_{\mathrm{s}}=16 a / 101$, uniformly filling the whole acoustic domain bounded by $S$.Figure 1 shows the distribution of the thresholded topological sensitivity $\hat{\mathcal{T}}$ defined by (4) in the three coordinate planes containing the true scatterer centroid $\boldsymbol{x}^{\text {true }} . \hat{\mathcal{T}}$ (and hence $\mathcal{T}$ ) is seen to attain its lowest values in zones corresponding to, or close to, the actual true scatterer location.
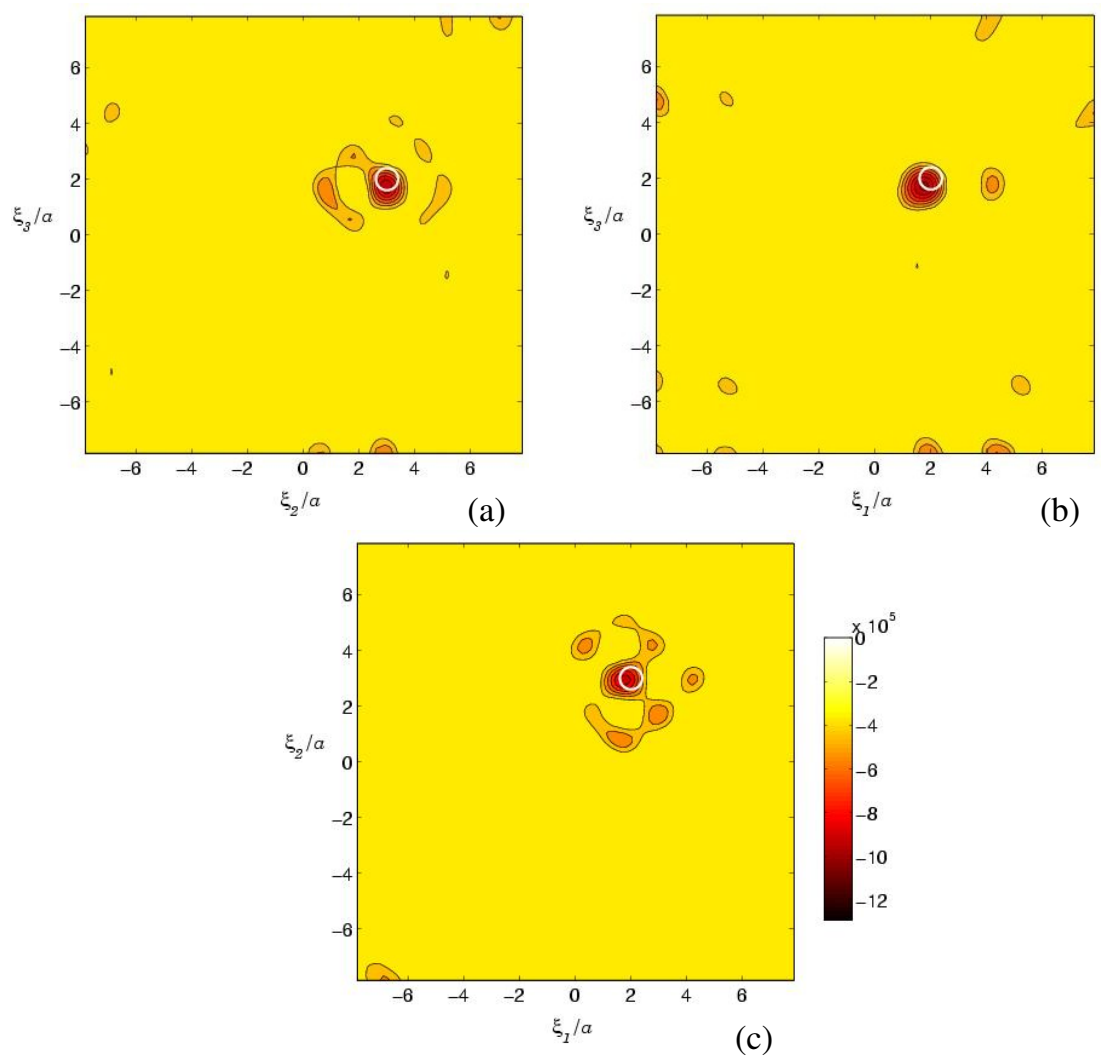

Figure 1. Identification of spherical hard scatterer of radius $0.4 a$ : distribution of thresholded topological sensitivity $\hat{\mathcal{T}}\left(\boldsymbol{x}_{s}\right)$ for sampling points $\boldsymbol{x}_{s}$ in coordinate planes $\xi_{1}=x_{1}^{\text {true }}(\boldsymbol{a}), x_{2}=x_{2}^{\text {true }}(\boldsymbol{b})$ and $x_{3}=x_{3}^{\text {true }}(\boldsymbol{c})$ 


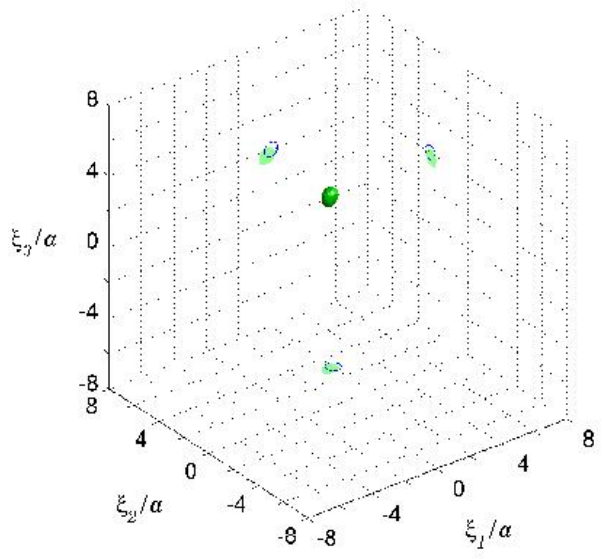

Figure 2. Identification of spherical hard scatterer of radius $0.4 a$ : iso-surfaces of $\mathcal{T}\left(\boldsymbol{x}_{s}\right)$ for $\mathcal{T}=0.55 \mathcal{T}_{\text {min }}$, computed on a truncated search grid such that $\left\{\left|\xi_{i}\right| \leq\right.$ $5 a(i=1,2,3)\}$. Values of $\mathcal{T}\left(\boldsymbol{x}_{s}\right)$ lower than the iso-value are inside the iso-surface

Table 2. CPU times and (in parentheses) GMRES iteration count for computing the true, free and adjoint solutions on the boundary, and CPU times for computing the topological sensitivity over the whole sampling grid

\begin{tabular}{|r||r|r|r||r|}
\hline & $u^{\text {true }}$ on $S \cup \Gamma^{\text {true }}$ & $u$ on $S$ & $\hat{u}$ on $S$ & $\mathcal{T}$ in $\Omega$ \\
\hline $2 L=16 a$ & $1444 \mathrm{~s}(435)$ & $969 \mathrm{~s}(282)$ & $1163 \mathrm{~s}(342)$ & $852 \mathrm{~s}$ \\
$2 L=32 a$ & $6461 \mathrm{~s}(439)$ & $5615 \mathrm{~s}(388)$ & $6818 \mathrm{~s}(476)$ & $1860 \mathrm{~s}$ \\
\hline
\end{tabular}

Figure 2 shows the iso-surfaces of the field $\mathcal{T}$ corresponding to $\mathcal{T}=0.55 \mathcal{T}_{\min }$, computed on a truncated sampling grid such that $\left\{\left|\xi_{i}\right| \leq 5 a(i=1,2,3)\right\}$, i.e. in which sampling points whose distance to $S$ is less than $3 a$ have been taken out. In this central region, low negative values of $\mathcal{T}$ occur only in the vicinity of the actual scatterer location. It should be mentioned that $\mathcal{T}$ has been observed to also achieve low values in zones located near edges or corners of $S$, where there is no scatterer.

For completeness, Table 2 provides typical CPU and iteration counts, for a cube of size $2 L=16 a$ as above and for a cube of size $2 L=32 a$ (the wavelength being kept at $\lambda=3 a$ ). It is interesting to observe that the overall CPU times for $\Omega(32 a)$, which involves roughly 4 times as many nodal unknowns as $\Omega(16 a)$, are about 5 times higher than those for $\Omega(16 a)$ (while the expected ratio for traditional BEM would be $\left.4^{3}=64\right)$, and that the GMRES iteration counts are only fractionally higher for $\Omega(32 a)$. These observations are consistent with the almost-linear $O(N \log N)$ complexity per iteration predicted by theoretical analyses of the FMM (Nishimura, 2002; Darve, 2000; Sylvand, 2003). All computations have been performed on a Linux $\mathrm{PC}$ computer with one $3 \mathrm{GHz}$ processor.

Similar computations featuring noisy data have also been performed. For cost functions that are quadratic with respect to measurement residuals, the adjoint field $\hat{u}$ 
undergoes perturbations that are proportional to the data noise. From [2], the perturbation induced on $\mathcal{T}_{3}$ therefore also depends linearly on the noise level. The present approach is therefore less sensitive to noise than conventional inversion techniques.

\section{Higher-order topological expansion}

The numerical experiments performed thus far clearly establish the usefulness of the topological sensitivity field $\mathcal{T}_{3}\left(\boldsymbol{x}_{\mathrm{s}}\right)$ as a defect indicator function. The information provided by $\mathcal{T}_{3}\left(\boldsymbol{x}_{\mathrm{s}}\right)$ is, however, of a qualitative nature only, as approximation [1] of $J$ at points $\boldsymbol{x}_{\mathrm{s}}$ such that $\mathcal{T}_{3}\left(\boldsymbol{x}_{\mathrm{s}}\right)<0$ is not amenable to minimization with respect to the obstacle size $\varepsilon$. One is hence led to consider expanding $J\left(\varepsilon, \boldsymbol{x}_{\mathrm{s}}\right)$ to higher order in $\varepsilon$ for the purpose of a subsequent minimization with respect to the obstacle size. As quadratic cost functions are often used in practice, such expansion should in view of [1] be carried out to order $O\left(\varepsilon^{6}\right)$, so as to square the $O\left(\varepsilon^{3}\right)$ leading order in [1]. This approach has for now been considered for the scalar Helmholtz equation (Bonnet, 2007), but can be extended to e.g. 3-D elastodynamics. The expansion of $J$ for any centrally-symmetric sound-hard scatterer, of characteristic size $\varepsilon$ and centered at $\boldsymbol{x}_{\mathrm{s}}$, is found to have the form

$$
J\left(\varepsilon, \boldsymbol{x}_{\mathrm{s}}\right)-J\left(0, \boldsymbol{x}_{\mathrm{s}}\right)=\underbrace{\varepsilon^{3} \mathcal{T}_{3}\left(\boldsymbol{x}_{\mathrm{s}}\right)+\varepsilon^{5} \mathcal{T}_{5}\left(\boldsymbol{x}_{\mathrm{s}}\right)+\varepsilon^{6} \mathcal{T}_{6}\left(\boldsymbol{x}_{\mathrm{s}}\right)}_{J_{6}\left(\varepsilon, \boldsymbol{x}_{\mathrm{s}}\right)}+o\left(\varepsilon^{6}\right)
$$

The previously known topological derivative $\mathcal{T}_{3}\left(\boldsymbol{x}_{\mathrm{s}}\right)$ and the new coefficients $\mathcal{T}_{5}\left(\boldsymbol{x}_{\mathrm{s}}\right), \mathcal{T}_{6}\left(\boldsymbol{x}_{\mathrm{s}}\right)$ have explicit expressions (not given hre for brevity) in terms of the acoustic Green's function associated with the reference acoustic domain and the structure of external boundary conditions. Due to the symmetry assumption for the small obstacle, there is no $O\left(\varepsilon^{4}\right)$ contribution in [5]. For cases where the Green's function is not known analytically, setting up $\mathcal{T}_{3}\left(\boldsymbol{x}_{\mathrm{s}}\right) \mathcal{T}_{5}\left(\boldsymbol{x}_{\mathrm{s}}\right), \mathcal{T}_{6}\left(\boldsymbol{x}_{\mathrm{s}}\right)$ entails solving boundary integral equations for (a) the forward and adjoint acoustic fields, and (b) auxiliary solutions generated by acoustic point sources at sampling points $\boldsymbol{x}_{\mathrm{s}}$. All of these solutions are governed by the same (integral) linear operator, associated with the Helmholtz equation in the reference (i.e. obstacle-free) domain, which makes the required supplementary computational work modest relative to the cost of one forward solution.

A simple approximate global search procedure. Expansions of the form [5] offer the option of minimizing the approximate polynomial expression $J_{6}\left(\varepsilon ; \boldsymbol{x}_{\mathrm{s}}\right)$ for sampling points chosen a priori. This task is, for each sampling point, simple and computationally very light. Hence, it can conceivably be performed for locations $\boldsymbol{x}_{\mathrm{s}}$ spanning a fine search grid $\mathcal{G}$, thereby defining an approximate global search procedure over the spatial region sampled by means of $\mathcal{G}$. The best estimate of the unknown scatterer $B^{\text {true }}$ yielded by this procedure is defined by the location $\boldsymbol{x}_{\mathrm{s}}=\boldsymbol{x}^{\text {est }}$ and size $\varepsilon=R^{\text {est }}$ leading to an absolute minimum of $J_{6}\left(\varepsilon ; \boldsymbol{x}_{\mathrm{s}}\right)$ over $\mathcal{G}$. In practice, a partial minimization of $J_{6}\left(\varepsilon ; \boldsymbol{x}_{\mathrm{s}}\right)$ w.r.t. $\varepsilon$ is performed for each $\boldsymbol{x}_{\mathrm{s}}$, and one defines the best estimate of the unknown scatterer over $\mathcal{G}$ in terms of its radius $R^{\text {est }}$ and center $\boldsymbol{x}^{\text {est }}$ by

$$
\left(R^{\text {est }}, \boldsymbol{x}^{\text {est }}\right)=\underset{\varepsilon>0, \boldsymbol{x}_{\mathrm{s}} \in \mathcal{G}}{\arg \min } J_{6}\left(\varepsilon, \boldsymbol{x}_{\mathrm{s}}\right)
$$




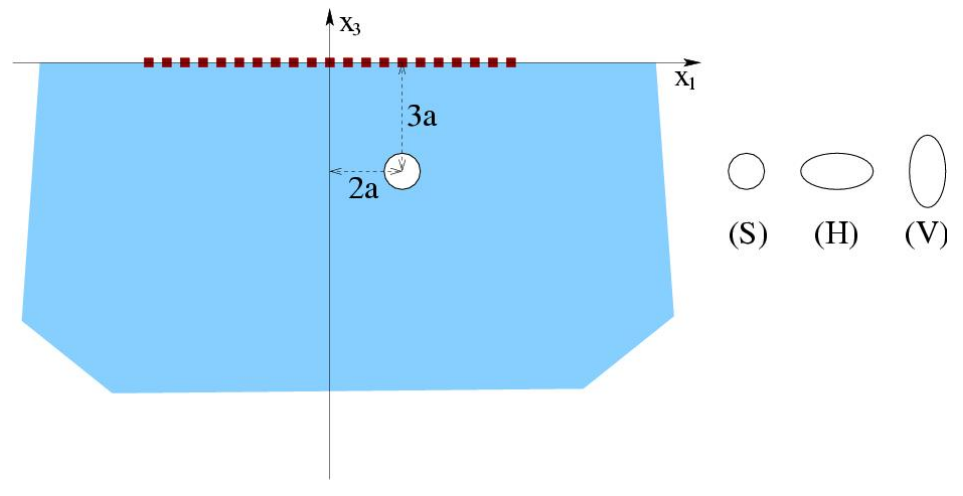

Figure 3. Identification of a spherical or ellipsoidal sound-hard scatterer in a acoustic half-space: geometry and notation

Numerical results. To demonstrate the proposed approximate search procedure and thereby demonstrate the usefulness of the $O\left(\varepsilon^{6}\right)$ expansion of $J\left(\varepsilon ; \boldsymbol{x}_{\mathrm{s}}\right)$, the identification of a hard scatterer embedded in an acoustic medium occupying the half-space $\Omega=\left\{\boldsymbol{\xi} \mid \xi_{3} \leq 0\right\}$ is considered, as depicted in Figure 3. A homogeneous Neumann condition is assumed on the surface $x_{3}=0$. Under these conditions, the relevant Green's function is, as mentioned earlier, explicitly known.

Three synthetic testing configurations are considered (hereinafter labelled $T^{(1)}$, $T^{(2)}, T^{(3)}$ ), where the square region $-5 a \leq \xi_{1}, \xi_{2} \leq 5 a$ on the surface $x_{3}=0$ is divided into $5 \times 5,10 \times 10$ and $20 \times 20$ squares, respectively ( $a$ being a reference length). Sensors are placed at all vertices $\boldsymbol{x}^{m}(1 \leq m \leq M)$ of the squares thus defined, so that $T^{(1)}, T^{(2)}, T^{(3)}$ feature $M=36,121,441$ points, respectively. Acoustic point sources of unit magnitude are placed in succession at all centers of the previously defined squares. The scattered field generated by each source is recorded at all sensors, so that $T^{(1)}, T^{(2)}, T^{(3)}$ support $N=25,100,400$ synthetic experiments, respectively. This is a situation of limited aperture, since data is available only on part of the surface of the half-space. The identification is performed on the basis of the leastsquares cost function

$$
\mathcal{J}\left(\Omega^{\star}\right)=\frac{1}{2} \sum_{q=1}^{N} \sum_{m=1}^{M}\left|u_{q}^{\text {obs }}\left(\boldsymbol{x}_{m}\right)-u_{q}^{\star}\left(\boldsymbol{x}_{m}\right)\right|^{2}
$$

where $u_{q}^{\text {obs }}$ and $u_{q}^{\star}$ denote the acoustic fields induced by a unit point source placed at $\boldsymbol{x}_{q}$ and for the 'true' and 'trial' configurations $\Omega^{\text {true }}=\Omega \backslash B^{\text {true }}$ and $\Omega^{\star}=\Omega \backslash B^{\star}$.

The approximate global search procedure has been performed on a search grid $\mathcal{G}$ of $51 \times 51 \times 25=65025$ regularly spaced sampling points spanning the 3 -D box-shaped region defined by $-10 a \leq x_{1}, x_{2} \leq 10 a,-10 a \leq x_{3} \leq-0.4 a$. The approximate cost function $J_{6}\left(\varepsilon ; \boldsymbol{x}_{\mathrm{s}}\right)$ with coefficients $\mathcal{T}_{3}\left(\boldsymbol{x}_{\mathrm{s}}\right), \mathcal{T}_{5}\left(\boldsymbol{x}_{\mathrm{s}}\right), \mathcal{T}_{6}\left(\boldsymbol{x}_{\mathrm{s}}\right)$ defined with reference 
Table 3. Radius estimate $R^{\text {est }}$ (best estimate over sampling grid $\mathcal{G}$ ) for testing configurations $T^{(1)}, T^{(2)}, T^{(3)}$ and noise-free synthetic data: sphere (a), horizontally elongated ellipsoid (b) and vertically elongated ellipsoid (c). A distance $\left\|\boldsymbol{x}_{s}^{\text {est }}-\boldsymbol{x}^{\text {true }}\right\|=$ $0.2 a$ was found in all cases

\begin{tabular}{|c|c|c|c|c|}
\hline & & \multicolumn{3}{|c|}{$R^{\text {est }} / R^{\text {true }}-1$} \\
\hline & & $T^{(1)}$ & $T^{(2)}$ & $T^{(3)}$ \\
\hline (S) & $\begin{aligned} k a & =0.5 \\
k a & =1 \\
k a & =2\end{aligned}$ & $\begin{array}{l}-4.18 \mathrm{e}-02 \\
-1.26 \mathrm{e}-01 \\
-3.33 \mathrm{e}-01\end{array}$ & $\begin{array}{l}-4.20 \mathrm{e}-02 \\
-1.26 \mathrm{e}-01 \\
-3.34 \mathrm{e}-01\end{array}$ & $\begin{array}{l}-4.20 \mathrm{e}-02 \\
-1.26 \mathrm{e}-01 \\
-3.34 \mathrm{e}-01\end{array}$ \\
\hline (H) & $\begin{aligned} k a & =0.5 \\
k a & =1 \\
k a & =2\end{aligned}$ & $\begin{array}{l}-2.31 \mathrm{e}-04 \\
-1.65 \mathrm{e}-01 \\
-4.22 \mathrm{e}-01\end{array}$ & $\begin{array}{r}9.05 \mathrm{e}-04 \\
-1.65 \mathrm{e}-01 \\
-4.22 \mathrm{e}-01\end{array}$ & $\begin{array}{r}8.89 \mathrm{e}-04 \\
-1.65 \mathrm{e}-01 \\
-4.22 \mathrm{e}-01\end{array}$ \\
\hline (V) & $\begin{aligned} k a & =0.5 \\
k a & =1 \\
k a & =2\end{aligned}$ & $\begin{array}{l}-7.42 \mathrm{e}-02 \\
-2.04 \mathrm{e}-01 \\
-4.64 \mathrm{e}-01\end{array}$ & $\begin{array}{l}-7.51 \mathrm{e}-02 \\
-2.06 \mathrm{e}-01 \\
-4.67 \mathrm{e}-01\end{array}$ & $\begin{array}{l}-7.51 \mathrm{e}-02 \\
-2.06 \mathrm{e}-01 \\
-4.67 \mathrm{e}-01\end{array}$ \\
\hline
\end{tabular}

to a small trial spherical scatterer, has been set up for all 65025 sampling points $\boldsymbol{x}_{\mathrm{s}} \in \mathcal{G}$ of the search grid thus defined and using the explicit Green's function.

The scatterer $B^{\text {true }}$ to be identified is centered at $\boldsymbol{x}^{\text {true }}=(2 a, 1.2 a,-3 a)$. Three geometries are considered for $B^{\text {true }}$ : a sphere (S) of radius $0.5 a$, a horizontally elongated ellipsoid $(\mathrm{H})$ with semiaxes $(a, 0.5 a, 0.5 a)$ and a vertically elongated ellipsoid (V) with semiaxes $(0.5 a, 0.5 a, a)$. For comparison purposes, a 'true' radius $R^{\text {true }}$ is defined for (S) by $R^{\text {true }}=0.5 a$ and for $(\mathrm{H})$ and $(\mathrm{V})$ by $R^{\text {true }}=2^{-2 / 3} a$, i.e. as the radius of the sphere having the same volume as $(\mathrm{H})$ or $(\mathrm{V})$. Three wavenumbers $k a=0.5,1,2$ have been considered. Note that $\boldsymbol{x}^{\text {true }} \notin \mathcal{G}$ : the sampling points closest to $\boldsymbol{x}^{\text {true }}$ are located above and below $\boldsymbol{x}^{\text {true }}$ and are separated from $\boldsymbol{x}^{\text {true }}$ by a vertical distance $0.2 a$.

The obstacle radius estimate $R^{\text {est }}$ (best estimate over sampling grid $\mathcal{G}$ ) obtained for the three frequencies considered and using the three testing configurations are compared to $R^{\text {true }}$ in table 3 , for the three geometries $(\mathrm{S}),(\mathrm{H})$ and $(\mathrm{V})$, using exact synthetic data. The lower-frequency case $k=0.5 a$ is seen to yield the most accurate estimation of $R^{\text {true }}$. In all cases, a distance $\left\|x_{\mathrm{s}}^{\text {est }}-\boldsymbol{x}^{\text {true }}\right\|=0.2 a$ is found, i.e. the grid point $\boldsymbol{x}_{\mathrm{s}}^{\text {est }}$ at which $J_{6}\left(\varepsilon ; \boldsymbol{x}_{\mathrm{s}}\right)$ achieves its lowest value $\hat{J}_{6}^{\min }$ is one of the two closest to $\boldsymbol{x}^{\text {true }}$ featured by the sampling grid $\mathcal{G}$. For the cases $(\mathrm{H})$ and $(\mathrm{V})$ where the shapes of the trial and true scatterers do not match, the 'equivalent radius' $R^{\text {true }}$ is nevertheless reasonably well estimated, and the location $\boldsymbol{x}_{\mathrm{s}}^{\text {est }}$ found is also optimal among the sites allowed by the chosen search grid. 
Here again, similar examples featuring simulated data noise have been considered. Numerical results for estimates $\left(R^{\text {est }}, \boldsymbol{x}^{\text {est }}\right)$ were only moderately affected by the noise, except in some of the cases where the coarsest testing configuration $T^{(1)}$ was used.

\section{Conclusions}

The approaches summarized herein are promising in that numerical experimentations performed thus far demonstrate their ability for approximate defect identification within computational costs of the order of a few forward solutions, i.e. substantially lower than that entailed by classical gradient-based "blind" minimization schemes and far lower than that required by global search algorithms. Moreover, they perform a search of global character through either the definition of a defect indicator function or the global minimization of an approximation to the original cost function. Their computational efficiency is further enhanced by resorting to fast multipole accelerated integral formulations.

Many theoretical and implementational issues remain open. The concept of topological sensitivity as defect indicator being heuristic, further theoretical study (possibly exploiting higher-order expansions) is desirable in order to e.g. better understand the conditions under which the approach can be a priori expected to perform well. Another open issue revolves around the identification of constitutive properties of inclusions from topological sensitivity formulations, for which promising preliminary results have been obtained by Guzina and Bonnet (2006) and Guzina and Chikichev (2007) but a complete analysis is still lacking. Extending the topological sensitivity to the case of vibratory measurements is also of interest, as formulations established so far are applicable only away from eigenfrequencies. Moreover, the issue of whether there are links between the present approach and that based on the distributed error in constitutive equation, which is also known from extensive numerical experiments to have the ability to (approximately) locate defects in structures (Ladeveze $e t$ al., 1993; Ladevèze and Chouaki, 1999; Bonnet and Reynier, 1998). Finally, from a computational standpoint, a finite element, discrete version of topological sensitivity might consist in considering perturbations of constitutive parameters (or nucleation of holes) over the scale of one finite element. Finally, applications to real data from e.g. NDT experiments sill remains to be done. Extensions to other physical contexts are currently under investigation, in particular in connection with infrared thermography.

\section{References}

Ammari H., Kang H., Reconstruction of small inhomogeneities from boundary measurements, Lecture Notes in Mathematics 1846, Springer-Verlag, 2004.

Bonnet M., "BIE and material differentiation applied to the formulation of obstacle inverse problems.”, Engng. Anal. with Bound. Elem., vol. 15, p. 121-136, 1995.

Bonnet M., Boundary Integral Equations Methods for Solids and Fluids, John Wiley and Sons, 1999a. 
Bonnet M., "Boundary element based formulations for crack shape sensitivity analysis", Engng. Anal. with Bound. Elem., vol. 25, p. 347-362, 2001.

Bonnet M., "Topological sensitivity for 3D elastodynamic and acoustic inverse scattering in the time domain", Comp. Meth. in Appl. Mech. Engng., vol. 195, p. 5239-5254, 2006.

Bonnet M., "Inverse acoustic scattering by small-obstacle expansion of misfit function", 2007, under review.

Bonnet M., Burczyński T., Nowakowski M., "Sensitivity analysis for shape perturbation of cavity or internal crack using BIE and adjoint variable approach", Int. J. Solids Struct., vol. 39, p. 2365-2385, 2002.

Bonnet M., Constantinescu A., "Inverse problems in elasticity", Inverse Problems, vol. 21, p. R1-R50, 2005. topical review article.

Bonnet M., Guzina B. B., "Sounding of finite solid bodies by way of topological derivative", Int. J. Num. Meth. in Eng., vol. 61, p. 2344-2373, 2004.

Bonnet M., Reynier M., "On the estimation of the geometrical support of modelling defects using the distributed error in constitutive equation.", Inverse Problems, Control and Shape Optimization, Carthage, Tunisie, p. 65-70, 1998.

Bui H. D., Fracture mechanics. Inverse problems and solutions, Springer-Verlag, 2006.

Chaillat S., Bonnet M., Semblat J. F., "A fast multipole accelerated BEM for 3-D elastic wave computation”, Revue Européenne de Mécanique Numérique, vol. 17, p. 701-712, 2008.

Colton D., Coyle J., Monk P., "Recent developments in inverse acoustic scattering theory.", SIAM Review, vol. 42, p. 369-414, 2000.

Colton D., Kirsch A., "A simple method for solving inverse scattering problems in the resonance region”, Inverse Problems, vol. 12, p. 383-393, 1996.

Darve E., "The fast multipole method: numerical implementation", J. Comp. Phys., vol. 160, p. 195-240, 2000.

Eschenauer H. A., Kobelev V. V., Schumacher A., "Bubble method for topology and shape optimization of structures", Structural Optimization, vol. 8, p. 42-51, 1994.

Farhat C., Tezaur R., Djellouli R., "On the solution of three-dimensional inverse obstacle acoustic scattering problems by a regularized Newton method", Inverse Problems, vol. 18, p. 1229-1246, 2002.

Feijóo G. R., "A new method in inverse scattering based on the topological derivative", Inverse Problems, vol. 20, p. 1819-1840, 2004.

Gallego R., Rus G., "Identification of cracks and cavities using the topological sensitivity boundsary integral equation”, Comp. Mech., vol. 33, p. 154-163, 2004.

Garreau S., Guillaume P., Masmoudi M., "The topological asymptotic for PDE systems: the elasticity case.”, SIAM J. Contr. Opt., vol. 39, p. 1756-1778, 2001.

Greengard L., Rokhlin V., "A new version of the fast multipole method for the Laplace equation in three dimensions", Acta Numerica, vol. 6, p. 229-270, 1997.

Guzina B. B., Bonnet M., "Topological derivative for the inverse scattering of elastic waves", Quart. J. Mech. Appl. Math., vol. 57, p. 161-179, 2004.

Guzina B. B., Bonnet M., "Small-inclusion asymptotic of misfit functionals for inverse problems in acoustics", IP, vol. 22, p. 1761-1785, 2006. 
Guzina B. B., Chikichev I., "From imaging to material identification: a generalized concept of topological sensitivity”, J. Mech. Phys. Solids, vol. 55, p. 245-279, 2007.

Guzina B. B., Nintcheu Fata S., Bonnet M., "On the stress-wave imaging of cavities in a semiinfinite solid”, Int. J. Solids Struct., vol. 40, p. 1505-1523, 2003.

Ladevèze P., Chouaki A., "Application of a posteriori error estimation for structural model updating", Inverse Problems, vol. 15, p. 49-58, 1999.

Ladeveze P., Reynier M., Nedjar D., "Parametric correction of finite element models using modal tests.", in H. D. B. M. Tanaka (ed.), Inverse problems in engineering mechanics, Springer-Verlag, p. 91-100, 1993.

Lambert M., Bonnet M., Lesselier D., "Introduction à la diffraction inverse en acoustique et élasticité”, in M. Bruneau, C. Potel (eds), Matériaux et acoustique, Hermes, p. 82-101, 2006.

Litman A., Lesselier D., Santosa F., "Reconstruction of a two-dimensional binary obstacle by controlled evolution of a level-set", Inverse Problems, vol. 14, p. 685-706, 1998.

Margonari M., Bonnet M., "Fast multipole method applied to the coupling of elastostatic BEM with FEM", Computers and Structures, vol. 83, p. 700-717, 2005.

Masmoudi M., Pommier J., Samet B., "The topological asymptotic expansion for the Maxwell equations and some applications", Inverse Problems, vol. 21, p. 547-564, 2005.

Michalewicz Z., Fogel D. B., How to solve it: modern heuristics, Springer-Verlag, 2004.

Nemitz N., Méthode multipôle rapide et sensibilité topologique pour l'identification approchée de défauts à partir de données de type acoustique, thèse de doctorat, Ecole Polytechnique, Palaiseau, France, 2006.

Nemitz N., Bonnet M., “Topological sensitivity and FMM-accelerated BEM applied to 3D acoustic inverse scattering", Engng. Anal. with Bound. Elem., 2007. (accepté).

Nintcheu Fata S., Guzina B. B., "A linear sampling method for near-field inverse problems in elastodynamics", Inverse Problems, vol. 20, p. 713-736, 2004.

Nishimura N., "Fast multipole accelerated boundary integral equation methods", Appl. Mech. Rev., vol. 55, p. 299-324, 2002.

Pommier J., Samet B., "The topological asymptotic for the Helmholtz equation with Dirichlet condition on the boundary of an arbitrarily shaped hole", SIAM J. Control Optim., vol. 43, p. 899-921, 2005.

Potthast R., "A survey on sampling and probe methods for inverse problems", Inverse Problems, vol. 22, p. R1-R47, 2006.

Samet B., Amstutz S., Masmoudi M., "The topological asymptotic for the Helmholtz equation”, SIAM J. Control Optim., vol. 42, p. 1523-1544, 2004.

Schumacher A., Topologieoptimierung von Bauteilstrukturen unter Verwendung von Lochpositionierungskriterien, thèse de doctorat, Univ. of Siegen, Germany, 1995.

Sokolowski J., Zochowski A., "On the topological derivative in shape optimization.", SIAM J. Control Optim., vol. 37, p. 1251-1272, 1999.

Sylvand G., "Complex industrial computations in electromagnetism using the fast multipole method", in G. Cohen, E. Heikkola, P. Joly, P. Neittaanmäki (eds), Mathematical and numerical methods aspects of wave propagation, Springer-Verlag, p. 657-662, 2003.

Tarantola A., Inverse problem theory and methods for model parameter estimation, SIAM, 2005. 